\title{
Hidradenitis Suppurativa in Kuala Lumpur, Malaysia: A 7-Year Retrospective Review
}

\author{
Moonyza Akmal Ahmad Kamil (iD) and Azura Mohd Affandi (D) \\ Department of Dermatology, Kuala Lumpur Hospital, Kuala Lumpur, Malaysia \\ Correspondence should be addressed to Moonyza Akmal Ahmad Kamil; moonyza@yahoo.com
}

Received 31 January 2018; Accepted 11 April 2018; Published 22 May 2018

Academic Editor: Markus Stucker

Copyright (C) 2018 Moonyza Akmal Ahmad Kamil and Azura Mohd Affandi. This is an open access article distributed under the Creative Commons Attribution License, which permits unrestricted use, distribution, and reproduction in any medium, provided the original work is properly cited.

\begin{abstract}
Introduction. Hidradenitis Suppurativa (HS) is a chronic inflammatory skin condition characterized by inflamed nodules, abscesses, sinus tracts, and scarring, which can occur in any skin containing folliculopilosebaceous units. We aim to identify the demographic and clinical characteristics and treatment modalities in patients with HS. Methods. A retrospective analysis involving records of patients diagnosed with HS in Hospital Kuala Lumpur from July 2009 to June 2016. Results. Sixty-two patients were identified, with equal cases involving males and females. Majority of patients were Malays (41.9\%), followed by Indians (35.5\%), Chinese (17.7\%), and other ethnicities (4.8\%). Median age at diagnosis was 25 (IQR: 14) years. There is a delay in diagnosis with a median of 24 (IQR: 52 ) months. Most of the patients had lesions on the axilla (85.5\%), followed by groin (33.9\%) and gluteal region (29\%). Gluteal lesions were more common in males. Nodules (67.7\%), sinuses (56.5\%), and abscesses (33.9\%) were the main clinical features, with 43.5\% classified under Hurley stage 2. There was no difference in terms of symptoms and types of lesions among different ethnicities and genders. Majority received systemic antibiotics, more than half had retinoid, and third of the patients had surgical intervention. Conclusions. A prompt recognition of HS is imperative, to screen for comorbidities and to initiate early treatment to reduce physical and psychological complications.
\end{abstract}

\section{Introduction}

Hidradenitis Suppurativa (HS), also known as Verneuil's disease, fox den disease, pyoderma fistulans significa (PFS), or acne inversa, has now been increasingly recognised to be due to a chronic inflammatory recurring skin condition that involves the follicular occlusion of the folliculopilosebaceous units (FPSUs) [1]. HS mainly affects the intertriginous skin areas of the axillary, groin, perianal, perineal, genital, and inframammary skin, as well as other areas of the skin which contains FPSUs. Follicular occlusion, follicular rupture, and the associated innate and adaptive immune dysregulation seem to be the essential events that lead to the development of HS. Bacterial infection and colonization are being regarded as secondary pathogenic factor that may worsen HS. Melnik and Plewig had proposed HS as an autoinflammatory disease characterized by dysregulation of gamma-secretase/Notch pathway [2]. Elevated levels of proinflammatory cytokines such as tumor necrosis factor- (TNF-) $\alpha$, interleukin- (IL-)
$1 \beta$, and IL-17 have been reported in HS lesions. Furthermore, there are also reports on genetic susceptibility, mechanical stresses on the skin, obesity, smoking, and hormonal factors which are closely related to HS [3].

The prevalence of HS is varied and not well described. It ranges from $0.05 \%$ to $6 \%$, depending on how and where the data were collected $[4,5]$. The most recent large population based study involving $>48$ million patients in United States found a much higher incidence of 11.4 per 100,000 population [6].

HS has a wide spectrum of clinical manifestation. It ranges from the relatively mild cases characterized by recurrent appearance of papules, pustules, and inflammatory nodules, to severe cases of deep fluctuant abscesses, draining sinuses, and keloidal scars. Although HS is not a lifethreatening condition, the pain, foul odour, and disfigurement are associated with significant decrease in quality of life [7]. The diagnosis of HS is made clinically but may be misdiagnosed especially by clinicians who are not familiar 
with this disease. In case of doubt, a skin biopsy may aid in excluding other diagnoses.

Hurley staging is frequently used to classify patients into 3 disease severity groups:

Stage I: abscess formation (single or multiple) without sinus tracts and scaring

Stage II: recurrent abscesses with sinus tracts and scarring, single, or multiple widely separated lesions

Stage III: diffuse or almost diffuse involvement, or multiple interconnected sinus tracts and abscesses across the entire area

HS is notoriously difficult to treat as it has multiple presentation and runs an unpredictable course. Treatment is based on disease severity, presence of comorbidities, patients' tolerance and preferability to the treatment, treatment cost, and the availability of treatment options. Besides the usual topical antibiotics and antiseptics dressings, there are systemic antibiotics, retinoids, and hormonal therapy. Recent evidence has shown that adalimumab and infliximab, 2 different monoclonal antibodies against TNF- $\alpha$, are effective in the treatment of moderate to severe HS. Adalimumab is the first Food and Drug Administration (FDA) approved treatment for moderate to severe HS in adults. The European Medicine Agency has also recently accepted HS as an indication for adalimumab. In 2015, the Board of the Italian Society of Dermatology and Venereology (SIDeMaST) has also given out guidelines on the role of TNF- $\alpha$ inhibitors in the management of HS [8]. Other trial drugs, such as IL-1 receptor antagonist (anakinra) and Human IgG kappa monoclonal antibody to IL-1 $\beta$ (canakinumab), have also shown improvement in HS $[9,10]$. Above all, patient education and psychological support play an important role in managing HS.

This study aims to identify the demographic and clinical characteristics and treatment modalities in patients with HS in Hospital Kuala Lumpur (HKL). We also compare the clinical characteristics of HS patients of different ethnicity and gender group.

\section{Materials and Methods}

This was a retrospective study involving all patients who had been clinically diagnosed with Hidradenitis Suppurativa from 1 July 2009 to 30 June 2016 in Dermatology Clinic, Kuala Lumpur Hospital. Based on the electronic record from the clinic registration, all of the clinic notes of these patients with the diagnosis of HS were traced and reviewed by 3 doctors from the clinic who had been trained to collect the data. Information such as the demographic information, clinical characteristics, and treatment modalities were extracted from patients' clinic notes and transcribed into the data collection form.

The collected data were analyzed using SPSS 22.0. Descriptive data were performed. All categorical variables such as gender and ethnicity, clinical characteristics, and treatment modalities were summarized as numbers and percentage, while continuous variables such as current age, age at diagnosis, and duration of illness before diagnosis were calculated and expressed in median and interquartile range
TABLE 1: Demographic characteristics of patients with HS.

\begin{tabular}{lcc}
\hline Characteristics & $n(\%)$ & Median (IQR) \\
\hline Gender & & \\
$\quad$ Male & $31(50)$ & \\
$\quad$ Female & $31(50)$ & \\
Ethnicity & $26(41.9)$ & \\
$\quad$ Malay & $22(35.5)$ & \\
$\quad$ Indian & $11(17.7)$ & \\
Chinese & $3(4.8)$ & \\
$\quad$ Others & & $31.5(14)$ \\
Current age (years old) & & $25.0(14)$ \\
Age at diagnosis (years old) & & $24.0(52)$ \\
$\begin{array}{l}\text { Duration of illness before } \\
\text { diagnosis (months) }\end{array}$ & & \\
\hline
\end{tabular}

(IQR). Assumption of normality was based on KolmogorovSmirnov statistics, skewness, kurtosis, histograms, and Q$\mathrm{Q}$ plots. The majority of our continuous variables were not normally distributed; therefore, nonparametric tests were used for further data analysis. The tests used were chi-square test for independence, Fisher's exact test, and Mann-Whitney $U$ test. A 2-tailed $p$ value of $<0.05$ is considered as statistically significant.

\section{Results}

Of the 47067 records of new cases registered in HKL Dermatology Clinic from 1 July 2009 to 30 June 2016, a total of 62 patients were identified to have the diagnosis of HS. This led to an incidence rate of 0.013 per 100,000 clinic population. An equal number of cases were seen between females and males. HS were seen in patients between 14 and 79 years old. The median age at diagnosis was 25 (IQR: 14 ) years. Male patients were noted to be diagnosed at an older age (median age: 33, IQR 16 years old) when compared to female patients (median age: 24 , IQR: 12 years old, $p=0.006$ ). There was no significant difference between both genders in the duration of illness before being diagnosed with HS ( $p=0.101)$.

Table 1 shows the demographic characteristics of patients with HS according to gender, ethnicity, age at diagnosis, and duration of illness before diagnosis. HS was more frequently seen in Malay (41.9\%), followed by Indian (35.5\%), Chinese (17.7\%), and other ethnicities (4.8\%). There was a delay in making the diagnosis with median duration of illness prior to diagnosis of 24 (IQR: 52) months, with the longest duration of 30 years. There were only 2 patients with similar family history of HS. Nine patients were cigarette smokers. Median BMI of our patients was 34.0 (IQR 20.2), calculated with missing data of up to $90 \%$.

Table 2 shows the clinical characteristics of our study cohort. Majority of the patients had lesions over the axilla (85.5\%), followed by groin (33.9\%) and gluteal region (29\%). Male patients had significant lesions over the gluteal region $(p=0.002)$ (Table 5).

The types of lesions commonly seen were nodules $(67.7 \%)$, sinuses $(56.5 \%)$, pustules $(37.1 \%)$, keloid scar $(35.5 \%)$, 
TABLE 2: Clinical characteristics of patients with HS.

\begin{tabular}{lc}
\hline Characteristics & $n(\%)$ \\
\hline Types of lesions & \\
Nodules & $42(67.7)$ \\
Sinus & $35(56.5)$ \\
Pustules & $23(37.1)$ \\
Keloid scar & $22(35.5)$ \\
Abscess & $21(33.9)$ \\
Double comedones & $2(3.2)$ \\
Fistula & $2(3.2)$ \\
Location & \\
Axilla & $53(85.5)$ \\
Groin & $21(33.9)$ \\
Gluteal & $18(29)$ \\
Chest & $10(16.1)$ \\
Back & $9(14.5)$ \\
Neck & $8(12.9)$ \\
Inframammary & $4(6.5)$ \\
Genitalia & $2(3.2)$ \\
Postauricular & $1(1.6)$ \\
Hurley staging & \\
1 & $22(35.5)$ \\
2 & $27(43.5)$ \\
3 & $13(21)$ \\
Symptoms & \\
Pain & $35(56.5)$ \\
Eruritus & $23(37.1)$ \\
\hline & $12(19.4)$ \\
\hline
\end{tabular}

and abscesses (33.9\%). More than half of them (56.5\%) reported symptoms of pain, while a third (37.1\%) reported pruritus. There was no significant difference detected in the type of lesions, severity, and symptoms of HS, in between different ethnicities, as well as between male and female patients (Tables 5 and 6)

About $35.5 \%$ of patients had positive swab or tissue culture, which grew a mixture of Gram-positive and Gramnegative organisms (Table 3 ).

In terms of treatment modalities, more than $75 \%$ of the patients had topical treatment, which include topical fusidic acid (30.6\%), mupirocin (8.1\%), clindamycin $(4.8 \%)$, and antiseptic wash such as chlorhexidine, prontosan, and octenisan wash $(66.1 \%)$. Fifty-two patients had received systemic antibiotics such as doxycycline (29\%), rifampicin (29\%), clindamycin $(21.1 \%)$, tetracycline $(8.1 \%)$, metronidazole $(6.5 \%)$, and minocycline $(1.6 \%)$. Thirty patients had isotretinoin while only 6 patients had acitretin. Six patients had dapsone for the treatment of HS. None of these patients had biologics. A third of these patients had some surgical intervention, which include incision and drainage (14 patients), localised excision (6 patients), and wide local excision and skin graft (3 patients). Figure 1 shows the treatment modalities of patients with HS.

Table 4 shows the comorbidities of the patients with HS. Seventeen patients (27.4\%) had Acne Conglobata or Nodulocystic Acne. Pilonidal sinus was diagnosed in 3 patients.
TABLE 3: Cultures taken from patients with HS.

\begin{tabular}{lc}
\hline Bacteriological culture (swab or tissue) & Number (\%) \\
\hline Gram-positive & $7(11.3)$ \\
Staphylococcus & $4(6.5)$ \\
MSSA & $3(4.8)$ \\
MRSA & $4(6.5)$ \\
GBS & $1(1.6)$ \\
Streptococcus pyogenes & $4(6.5)$ \\
Diphtheroids & $1(1.6)$ \\
Enterococcus & \\
Gram-negative & $6(9.7)$ \\
E. coli & $5(8.1)$ \\
Proteus sp. & $4(6.5)$ \\
Klebsiella pneumonia & $2(3.2)$ \\
Acinetobacter & $7(11.3)$ \\
Mixed growth & \\
Others & $1(1.6)$ \\
$\quad$ Chrysosporium sp. & MSSA: Methicillin Sensitive Staphylococcus aureus, MRSA: Methicillin \\
Resistant Staphylococcus aureus, and GBS: Group B Streptococcus.
\end{tabular}

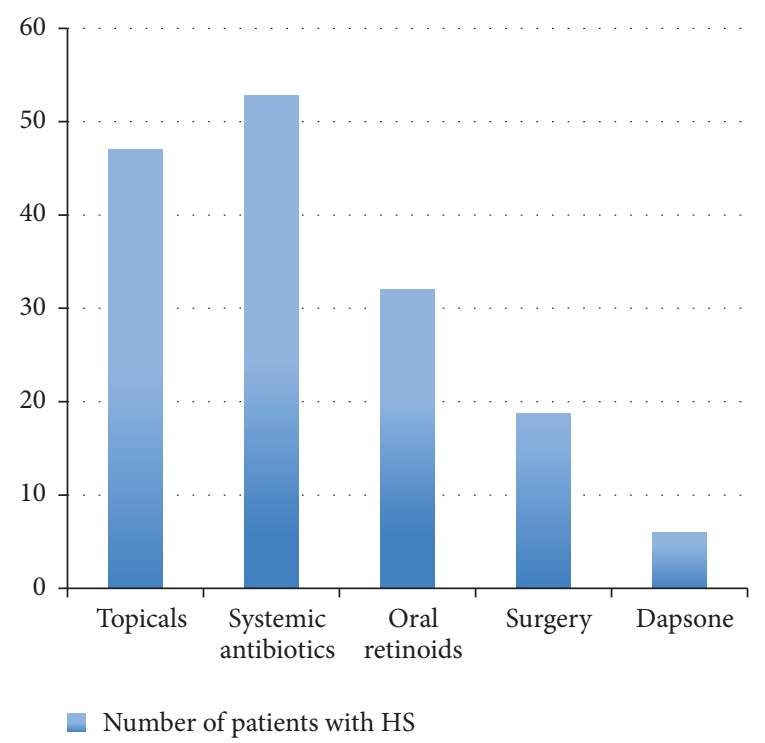

FIgURE 1: Treatment modalities of patients with HS.

None of these patients fulfilled the criteria of the follicular occlusion triad or tetrad, only 9 patients $(14.5 \%)$ with obesity, 6 patients $(9.7 \%)$ with diabetes mellitus, and 4 (6.5\%) with hypertension and dyslipidemia. One patient had concurrent HIV and hepatitis C infection. Depression was also detected in 1 patient with HS. In Table 6, HS patients with concurrent hypertension and dyslipidemia are seen more in the Chinese population ( $p=0.039, p=0.031$ consecutively).

\section{Discussion}

Sixty-two patients had the diagnosis of HS from the review of medical records from 1 July 2009 to 30 June 2016. This has shown an increasing trend when we compare to a similar 
TABLE 4: Comorbidities in patients with HS.

\begin{tabular}{lc}
\hline Comorbidities & Number (\%) \\
\hline Acne conglobata/nodulocystic & $17(27.4)$ \\
Obesity & $9(14.5)$ \\
Diabetes & $6(9.7)$ \\
Hypertension & $4(6.5)$ \\
Dyslipidemia & $4(6.5)$ \\
Pilonidal sinus & $3(4.8)$ \\
Anemia & $3(4.8)$ \\
Neoplasm (SCC) & $1(1.6)$ \\
RVD and hepatitis C & $1(1.6)$ \\
Depression & $1(1.6)$ \\
\hline
\end{tabular}

study done by Leelavathi et al. which reported only 15 patients over 5-year period [11] (Table 7).

Many studies reported that HS is a rarely diagnosed disease [4, 12, 13]. Vazquez et al. reported that only 268 patients were diagnosed with HS in Minnesota between 1968 and 2008, with an overall annual age- and sex-adjusted incidence of 6.0 per 100,000. Cosmatos et al. found a low rate of clinically detected HS where the overall prevalence estimate was $0.053 \%$ in a retrospective analysis using health insurance database in the United States (Table 7). We found an incidence rate of 0.013 in 100,000 clinic population, which is much lower than previous studies. This is because Hospital Kuala Lumpur, being a fully subsidized government hospital, is a major tertiary center in Malaysia. Although primarily covering Kuala Lumpur and its surrounding area, it also has patients coming from other parts of the country, including almost the whole of Peninsular Malaysia.

We noted that the ratio of male to female patients was equal, which is inconsistent with previous studies [4, 5, 13-15]. Our number of male to female patients visits to dermatology clinic during this study period was almost equal (1:0.94). Shali Alikhan et al. reported that women outnumbered men with HS by nearly 3 to 1 , and Garg et al. reported that prevalence of female patients was more than 2 -fold greater than in male patients. The only study that reported an obvious male predominant in HS was seen in a study in Tunisia [16].

In our study, the median age at diagnosis was 25 (IQR: 14) years. Male patients with HS were found to be significantly older at the time of diagnosis compared to female patients, with median age of 33, IQR 16 years old for males, compared to median age of 24, IQR 12 years old for females, with $p=$ 0.006 . This is in line with a study in Minnesota which found that the highest incidence of HS is among young women aged 20-29 (18.4 per 100,000) [4]. Cosmatos et al. [13] found that most patients with HS were aged 30 to 64 years (Table 7).

The diagnosis of HS is often delayed. This is due to the fact that HS has a wide spectrum of manifestation and may mimic other illnesses and commonly treated as skin infections such as recurrent furuncles. This delay may be caused by patient delay in seeking treatment, the clinician not making the correct diagnosis, or both. The longest duration of diagnosis after the onset of symptoms in this study was 30 years. This patient had gone to multiple primary care physicians who had failed to give the accurate diagnosis. We also had a patient who had underwent several surgical interventions to the abscesses and only later diagnosed with HS. This finding is in line with previous studies, which reported significant delay in the diagnosis of HS $[4,17]$.

It was also surprising to see that, in our study, the proportion of Indians diagnosed with HS was much higher (35.5\%) when compared with the number of new cases of Indian patients during the study period (18.1\%). A study by McMillan in 2014 reported that the percentage of visits with HS diagnosis in black patients was significantly higher than the percentage of all visits by black patients in a combined data from the National Ambulatory Medical Care Survey (NAMCS) and National Hospital Ambulatory Medical Care Survey (NHAMCS) in the United States [12]. In another study, the adjusted HS prevalence among African American patients with HS was more than 3-fold than that among white patients [15]. An increased frequency of HS is observed in blacks, possibly because blacks have a greater density of apocrine glands than whites. This same explanation can be postulated in our finding associating HS with the Indian ethnicity in Malaysia, who has darker Fitzpatrick phototype as compared to other ethnicities such as Malay and Chinese.

Although there are emerging data on the association of obesity, metabolic syndrome, and other related comorbidities with HS [18-20], we did not find significant difference statistically between obesity and metabolic syndrome with HS. This is due to the limited documentation in our retrospective study. Missing data were up to $90 \%$ in certain categories, as they were not documented in patients' record.

However, with the limited data, we noted that Chinese patients had significantly higher percentage of having concurrent hypertension and dyslipidemia, but there was no difference of comorbidities in between males and females. With the combination of smoking in patients with HS, these contribute to a higher risk of cardiovascular-associated death, as shown in Danish HS patients [21]. None of our patients reported having inflammatory bowel disease or rheumatology condition, as described previously. Previous studies have found that patients with HS have a higher risk of developing depression [22]. In our study, only 1 patient had been diagnosed with depression and currently being treated by the psychiatry team. The low prevalence of depression may be due to the fact that psychiatry illness is still a taboo in our country and therefore patients do not openly express their emotional distress to the clinician.

Majority of the patients had lesions over the axilla, followed by groin and gluteal region, inframammary, chest, back, neck, and postauricular region. The lesions were composed of nodules, sinuses, pustules, keloid scar, and abscesses. At diagnosis, majority of the patients had moderate to severe symptoms (Hurley stages 2 and 3 ) and more than half of them reported symptoms of pain, while a third reported pruritus. Previous studies had reported that the distribution of lesions in HS is influence by gender. Primary sites of involvement in females are the groin or upper inner thigh, axilla, upper anterior torso (including breast and inframammary regions), and the buttocks or gluteal clefts [4, 23]. In males, the common sites are the groin or thigh, axilla, perineal or 
TABLE 5: Comparison of HS clinical characteristics between male and female patients with HS.

\begin{tabular}{|c|c|c|c|}
\hline Clinical characteristics & $\begin{array}{c}\text { Male }(n=31) \\
n(\%)\end{array}$ & $\begin{array}{c}\text { Female }(n=31) \\
n(\%)\end{array}$ & $p$ value \\
\hline \multicolumn{4}{|l|}{ Severity of $H S$} \\
\hline Mild (Hurley stage 1) & $8(25.8)$ & $14(45.2)$ & \multirow{2}{*}{0.111} \\
\hline Moderate to severe (Hurley stage 2-3) & $23(74.2)$ & $17(54.8)$ & \\
\hline \multicolumn{4}{|l|}{ Symptoms } \\
\hline Embarrassment & $4(12.9)$ & $0(0)$ & 0.113 \\
\hline Pain & $18(58.1)$ & $17(54.8)$ & 0.798 \\
\hline Pruritus & $11(35.5)$ & $12(38.7)$ & 0.793 \\
\hline \multicolumn{4}{|l|}{ Location of lesion } \\
\hline Axilla & $25(80.6)$ & $28(90.3)$ & 0.279 \\
\hline Groin & $9(29.0)$ & $12(38.7)$ & 0.421 \\
\hline Gluteal & $15(48.4)$ & $3(9.7)$ & 0.002 \\
\hline Chest & $4(12.9)$ & $6(19.4)$ & 0.490 \\
\hline Back & $7(22.6)$ & $2(6.5)$ & 0.071 \\
\hline Neck & $2(6.5)$ & $6(19.4)$ & 0.130 \\
\hline Inframammary & $2(6.5)$ & $2(6.5)$ & 1.000 \\
\hline Genitalia & $1(3.2)$ & $1(3.2)$ & 1.000 \\
\hline Postauricular & $1(3.2)$ & $0(0)$ & 0.313 \\
\hline \multicolumn{4}{|l|}{ Types of lesion } \\
\hline Papules & $8(25.8)$ & $7(22.6)$ & 0.767 \\
\hline Nodules & $22(71.0)$ & $20(64.5)$ & 0.587 \\
\hline Sinus & $19(61.3)$ & $16(51.6)$ & 0.442 \\
\hline Pustules & $12(38.7)$ & $11(35.5)$ & 0.793 \\
\hline Keloid scar & $13(41.9)$ & $9(29.0)$ & 0.288 \\
\hline Abscess & $12(38.7)$ & $9(29.0)$ & 0.421 \\
\hline Double comedones & $1(3.2)$ & $1(3.2)$ & 1.000 \\
\hline Fistula & $1(3.2)$ & $1(3.2)$ & 1.000 \\
\hline \multicolumn{4}{|l|}{ Comorbidities } \\
\hline Nodulocystic acne & $9(29.0)$ & $8(25.8)$ & 0.776 \\
\hline Obesity & $4(12.9)$ & $5(16.1)$ & 0.718 \\
\hline Diabetes & $4(12.9)$ & $2(6.5)$ & 0.671 \\
\hline Hypertension & $3(9.7)$ & $1(3.2)$ & 0.612 \\
\hline Dyslipidemia & $2(6.5)$ & $2(6.5)$ & 1.000 \\
\hline
\end{tabular}

perianal regions, and buttocks or gluteal cleft [24]. However, when comparing between males and females in our patients with HS, there was no significant difference between the severity, symptoms of HS, or the location of lesions, except that male patients had more significant lesions over the gluteal region.

Although routine bacterial culture is not indicated, we noted that patients with secondary bacterial infection worsened HS condition. In this study, the swab and/or tissue culture grew mixture of Gram-positive and Gram-negative organism, which can aid in the choice of antibiotics for these patients.

The most common oral tetracycline regime in our clinic is doxycycline, given in the highest percentage of patients, at the dosage of $100 \mathrm{mg}$ once to twice daily. This is the key treatment for mild to moderate HS with favourable adverse effect profile. Combination therapy with clindamycin and rifampicin is the next option, usually to those who have failed oral tetracycline. Our regimen of combination therapy is clindamycin $300 \mathrm{mg}$ twice daily and rifampicin $300 \mathrm{mg}$ twice daily. Previous studies have shown that $35 \%-70 \%$ of patients had at least some improvement in their disease course, with sustained efficacy of more than $40 \%$ in 1 year [25-27].

Besides systemic antibiotics, more than half of our cohort received oral retinoid. Most of these patients were on isotretinoin, especially those with concurrent nodulocystic acne. Studies involving acitretin treatment have reported significant improvements in up to $60 \%$ of patients [27]. It is also more effective when used as an adjuvant to other systemic medications. Surgical interventions were also treatment choice for those with recurrent and complicated HS. 
TABLE 6: Comparison of clinical characteristics of HS among different ethnicity group.

\begin{tabular}{|c|c|c|c|c|c|c|}
\hline Clinical characteristics & $\begin{array}{c}\text { Malay }(n=26) \\
n(\%)\end{array}$ & $\begin{array}{c}\text { Chinese }(n=11) \\
n(\%)\end{array}$ & $\begin{array}{c}\text { Indian }(n=22) \\
n(\%) \\
\end{array}$ & $\begin{array}{c}\text { Others }(n=3) \\
n(\%)\end{array}$ & Total $(n=62)$ & $p$ value \\
\hline \multicolumn{7}{|l|}{ Severity of HS } \\
\hline Mild & $9(34.6)$ & $3(27.3)$ & $9(40.9)$ & $1(33.3)$ & $22(35.5)$ & \multirow{2}{*}{0.892} \\
\hline Moderate-severe & $17(65.4)$ & $8(72.7)$ & $13(59.1)$ & $2(66.7)$ & $40(64.5)$ & \\
\hline \multicolumn{7}{|l|}{ Symptoms } \\
\hline Pain & $14(53.8)$ & $4(36.4)$ & $15(68.2)$ & $2(66.7)$ & $35(56.5)$ & 0.357 \\
\hline Pruritus & $8(30.8)$ & $3(27.3)$ & $10(45.5)$ & $2(66.7)$ & $23(37.1)$ & 0.443 \\
\hline Embarrassment & $1(3.8)$ & $1(9.1)$ & $1(4.5)$ & $1(33.3)$ & $12(19.4)$ & 0.246 \\
\hline \multicolumn{7}{|l|}{ Location of lesion } \\
\hline Axilla & $23(88.5)$ & $6(54.5)$ & $21(95.5)$ & $3(100)$ & $53(85.5)$ & 0.022 \\
\hline Groin & $7(26.9)$ & $6(54.5)$ & $7(31.8)$ & $1(33.3)$ & $21(33.9)$ & 0.440 \\
\hline Gluteal & $6(23.1)$ & $7(63.6)$ & $5(22.7)$ & $0(0)$ & $18(29)$ & 0.061 \\
\hline Chest & $5(19.2)$ & $0(0)$ & $5(22.7)$ & $0(0)$ & $10(16.1)$ & 0.310 \\
\hline Back & $4(15.4)$ & $4(36.4)$ & $1(4.5)$ & $0(0)$ & $9(14.5)$ & 0.089 \\
\hline Neck & $4(15.4)$ & $2(18.2)$ & $2(9.1)$ & $0(0)$ & $8(12.9)$ & 0.766 \\
\hline Inframammary & $3(11.5)$ & $0(0)$ & $0(0)$ & $1(33.3)$ & $4(6.5)$ & 0.072 \\
\hline Genitalia & $1(3.8)$ & $0(0)$ & $1(4.5)$ & $0(0)$ & $2(3.2)$ & 0.892 \\
\hline Postauricular & $1(3.8)$ & $0(0)$ & $0(0)$ & $0(0)$ & $1(1.6)$ & 0.704 \\
\hline \multicolumn{7}{|l|}{ Types of lesion } \\
\hline Nodules & $18(69.2)$ & $8(72.7)$ & $15(68.2)$ & $1(33.3)$ & $42(67.7)$ & 0.620 \\
\hline Sinus & $14(53.8)$ & $8(72.7)$ & $11(50.0)$ & $2(66.7)$ & $35(56.5)$ & 0.624 \\
\hline Pustules & $6(23.1)$ & $6(54.5)$ & $9(40.9)$ & $2(66.7)$ & $23(37.1)$ & 0.180 \\
\hline Keloid scar & $9(34.6)$ & $6(54.5)$ & $7(31.8)$ & $0(0)$ & $22(35.5)$ & 0.316 \\
\hline Abscess & $7(26.9)$ & $4(36.4)$ & $8(36.4)$ & $2(66.7)$ & $21(33.9)$ & 0.553 \\
\hline Double comedones & $0(0)$ & $0(0)$ & $1(4.5)$ & $1(33.3)$ & $2(3.2)$ & 0.083 \\
\hline Fistula & $2(7.7)$ & $0(0)$ & $0(0)$ & $0(0)$ & $2(3.2)$ & 0.413 \\
\hline \multicolumn{7}{|l|}{ Comorbidities } \\
\hline Acne conglobata/nodulocystic & $8(30.8)$ & $3(27.3)$ & $6(27.3)$ & $0(0)$ & $17(27.4)$ & 0.734 \\
\hline Obesity & $5(19.2)$ & $1(9.1)$ & $3(13.6)$ & $0(0)$ & $9(14.5)$ & 0.741 \\
\hline Diabetes & $3(11.5)$ & $2(18.2)$ & $0(0)$ & $1(33.3)$ & $6(9.7)$ & 0.152 \\
\hline Hypertension & $1(3.8)$ & $3(27.3)$ & $0(0)$ & $0(0)$ & $4(6.5)$ & 0.039 \\
\hline Dyslipidemia & $1(3.8)$ & $2(18.2)$ & $0(0)$ & $1(33.3)$ & $4(6.5)$ & 0.031 \\
\hline
\end{tabular}

Although recent studies have shown that monoclonal antibodies against TNF- $\alpha$ are effective in moderate to severe HS, none of our patients had yet to receive biologic treatment [28]. This is due to our limited financial resources in this fully funded government clinic. Perhaps when the generic version of biologics or "biosimilars" is widely available, and cost is not an issue anymore, more patients would be on biologic treatment especially those with recalcitrant HS.

We had difficulty in ascertaining the outcome of treatment, as most of them had received multiple treatment modalities in their course of management. Furthermore, we noted that about half of these patients had defaulted on their appointment. This is the limitation that needs to be addressed as this study relied on previous documentations on patients' medical record. In addition, our result may have limited generalizability, as the population size is very small.
Therefore, a larger, prospective cohort study is recommended to look specifically at the treatment modalities and the outcome of each treatment in the near future.

\section{Conclusion}

In conclusion, there is an equal gender distribution in our HS patients, with higher proportion in Indian population. Most of our patients were young adults, with a median age of diagnosis of 25 years, with long delay in the diagnosis of HS. Thus, it is pertinent that clinicians should be able to make an accurate diagnosis with comprehensive clinical examination, to screen for the associated comorbidities and to initiate treatment early to avoid the physical and emotional complications of this debilitating chronic skin disease. 


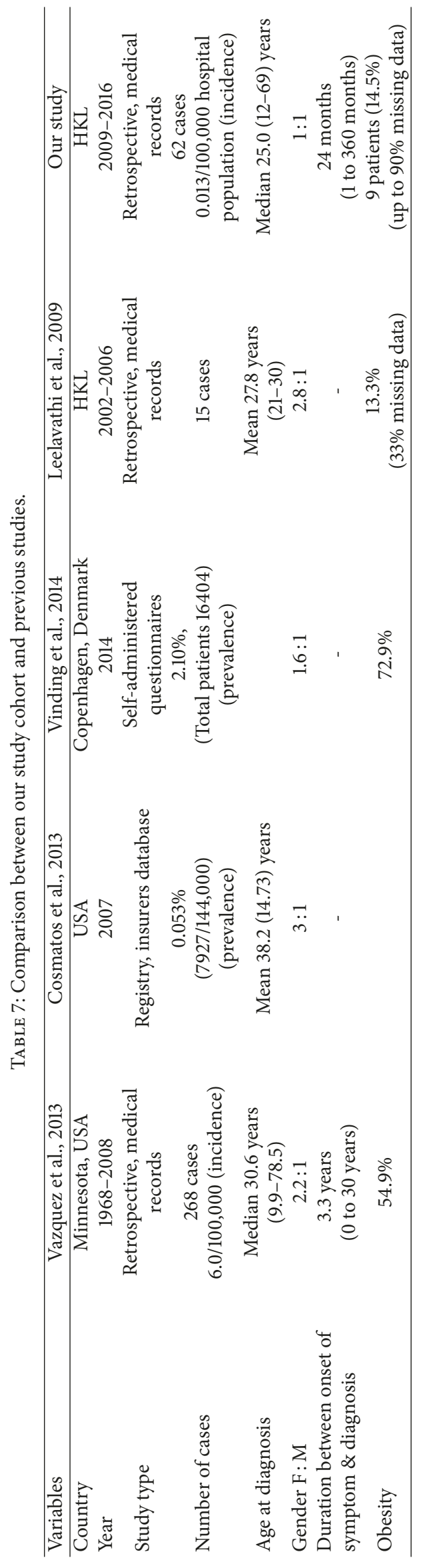




\section{Data Availability}

The datasets generated during and/or analysis in this study is available from the corresponding author on reasonable request.

\section{Disclosure}

This was a self-funded study and had received no outsourced funds.

\section{Conflicts of Interest}

The authors declare that there are no conflicts of interest regarding the publication of this article.

\section{Acknowledgments}

The authors would like to thank the Director General of Health Malaysia for his permission to publish this article. The authors would also like to express their gratitude to Dr. Ong Sian Yen and Dr. Kanimoli Rathakrishnan for helping in data collection.

\section{References}

[1] H. H. van der Zee, J. D. Laman, J. Boer, and E. P. Prens, "Hidradenitis suppurativa: viewpoint on clinical phenotyping, pathogenesis and novel treatments," Experimental Dermatology, vol. 21, no. 10, pp. 735-739, 2012.

[2] B. C. Melnik and G. Plewig, "Impaired Notch-MKP-1 signalling in hidradenitis suppurativa: An approach to pathogenesis by evidence from translational biology," Experimental Dermatology, vol. 22, no. 3, pp. 172-177, 2013.

[3] M. Napolitano, M. Megna, E. A. Timoshchuk et al., "Hidradenitis suppurativa: From pathogenesis to diagnosis and treatment," Clinical, Cosmetic and Investigational Dermatology, vol. 10, pp. 105-115, 2017.

[4] B. G. Vazquez, A. Alikhan, A. L. Weaver, D. A. Wetter, and M. D. Davis, "Incidence of hidradenitis suppurativa and associated factors: a population-based study of Olmsted County, Minnesota," Journal of Investigative Dermatology, vol. 133, no. 1, pp. 97-103, 2013.

[5] G. R. Vinding, I. M. Miller, K. Zarchi, K. S. Ibler, C. Ellervik, and G. B. E. Jemec, "The prevalence of inverse recurrent suppuration: A population-based study of possible hidradenitis suppurativa," British Journal of Dermatology, vol. 170, no. 4, pp. 884-889, 2014.

[6] A. Garg et al., "Incidence of hidradenitis suppurativa in the United States: A sex- and age-adjusted population analysis," Journal of the American Academy of Dermatology, vol. 77, no. 1, pp. 118-122, 2017.

[7] Ł. Matusiak, A. Bieniek, and J. C. Szepietowski, "Psychophysical aspects of hidradenitis suppurativa," Acta DermatoVenereologica, vol. 90, no. 3, pp. 264-268, 2010.

[8] M. Megna et al., "Hidradenitis suppurativa: guidelines of the Italian Society of Dermatology and Venereology (SIDeMaST) for the use of anti-TNF-alpha agents," Giornale Italiano di Dermatologia e Venereologia, vol. 150, no. 6, pp. 731-739, 2015.
[9] V. Tzanetakou et al., "Safety and Efficacy of Anakinra in Severe Hidradenitis Suppurativa: A Randomized Clinical Trial," JAMA Dermatol, vol. 152, no. 1, pp. 52-59, 2016.

[10] C. Houriet, S. M. Seyed Jafari, R. Thomi et al., "Canakinumab for severe hidradenitis suppurativa: Preliminary experience in 2 cases," JAMA Dermatology, vol. 153, no. 11, pp. 1195-1197, 2017.

[11] Leelavathi M. et al., "Hidradenitis Suppurativa: A Review of 15 Patients," Malaysian Journal of Dermatology, vol. 23, p. 10, 2009.

[12] K. McMillan, "Hidradenitis suppurativa: Number of diagnosed patients, demographic characteristics, and treatment patterns in the United States," American Journal of Epidemiology, vol. 179, no. 12, pp. 1477-1483, 2014.

[13] I. Cosmatos, A. Matcho, R. Weinstein, M. O. Montgomery, and P. Stang, "Analysis of patient claims data to determine the prevalence of hidradenitis suppurativa in the United States," Journal of the American Academy of Dermatology, vol. 68, no. 3, pp. 412-419, 2013.

[14] A. Alikhan, P. J. Lynch, and D. B. Eisen, "Hidradenitis suppurativa: a comprehensive review," Journal of the American Academy of Dermatology, vol. 60, no. 4, pp. 539-561, 2009.

[15] A. Garg, J. S. Kirby, J. Lavian, G. Lin, and A. Strunk, "Sexand age-adjusted population analysis of prevalence estimates for hidradenitis suppurativa in the United States," JAMA Dermatology, vol. 153, no. 8, pp. 760-764, 2017.

[16] A. Mebazaa et al., "Hidradenitis suppurativa: a disease with male predominance in Tunisia," Acta Dermatovenerol Alp Pannonica Adriat, vol. 18, no. 4, pp. 165-172, 2009.

[17] R. Simpson, "Diagnostic delay in hidradenitis suppurativa is a global problem," British Journal of Dermatology, vol. 173, no. 6, pp. 1546-1549, 2015.

[18] V. Bettoli, L. Naldi, S. Cazzaniga et al., "Overweight, diabetes and disease duration influence clinical severity in hidradenitis suppurativa-acne inversa: evidence from the national Italian registry," British Journal of Dermatology, vol. 174, no. 1, pp. 195197, 2016.

[19] I. M. Miller, C. Ellervik, G. R. Vinding et al., "Association of metabolic syndrome and hidradenitis suppurativa," JAMA Dermatology, vol. 150, no. 12, pp. 1273-1280, 2014.

[20] J. Shlyankevich, A. J. Chen, G. E. Kim, and A. B. Kimball, "Hidradenitis suppurativa is a systemic disease with substantial comorbidity burden: A chart-verified case-control analysis," Journal of the American Academy of Dermatology, vol. 71, no. 6, pp. 1144-1150, 2014.

[21] A. Egeberg, G. H. Gislason, and P. R. Hansen, "Risk of major adverse cardiovascular events and all-cause mortality in patients with hidradenitis suppurativa," JAMA Dermatology, vol. 152, no. 4, pp. 429-434, 2016.

[22] E. Shavit, J. Dreiher, T. Freud, S. Halevy, S. Vinker, and A. D. Cohen, "Psychiatric comorbidities in 3207 patients with hidradenitis suppurativa," Journal of the European Academy of Dermatology and Venereology, vol. 29, no. 2, pp. 371-376, 2015.

[23] J. Revuz, "Hidradenitis suppurativa," Journal of the European Academy of Dermatology and Venereology, vol. 23, no. 9, pp. 985-998, 2009.

[24] A. M. R. Schrader, I. E. Deckers, H. H. Van Der Zee, J. Boer, and E. P. Prens, "Hidradenitis suppurativa: A retrospective study of 846 Dutch patients to identify factors associated with disease severity," Journal of the American Academy of Dermatology, vol. 71, no. 3, pp. 460-467, 2014.

[25] H. H. Van Der Zee, J. Boer, E. P. Prens, and G. B. E. Jemec, "The effect of combined treatment with oral clindamycin 
and oral rifampicin in patients with hidradenitis suppurativa," Dermatology, vol. 219, no. 2, pp. 143-147, 2009.

[26] V. Bettoli, S. Zauli, A. Borghi et al., "Oral clindamycin and rifampicin in the treatment of hidradenitis suppurativa-acne inversa: A prospective study on 23 patients," Journal of the European Academy of Dermatology and Venereology, vol. 28, no. 1, pp. 125-126, 2014.

[27] J. Boer and M. Nazary, "Long-term results of acitretin therapy for hidradenitis suppurativa. Is acne inversa also a misnomer?" British Journal of Dermatology, vol. 164, no. 1, pp. 170-175, 2011.

[28] N. Scuderi, A. Monfrecola, L. A. Dessy, G. Fabbrocini, M. Megna, and G. Monfrecola, "Medical and Surgical Treatment of Hidradenitis Suppurativa: A Review," Skin Appendage Disorders, vol. 3, no. 2, pp. 95-110, 2017. 


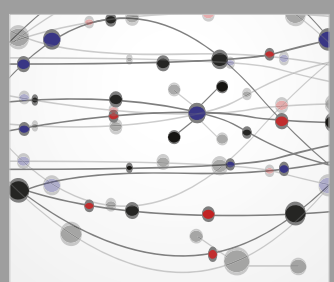

The Scientific World Journal
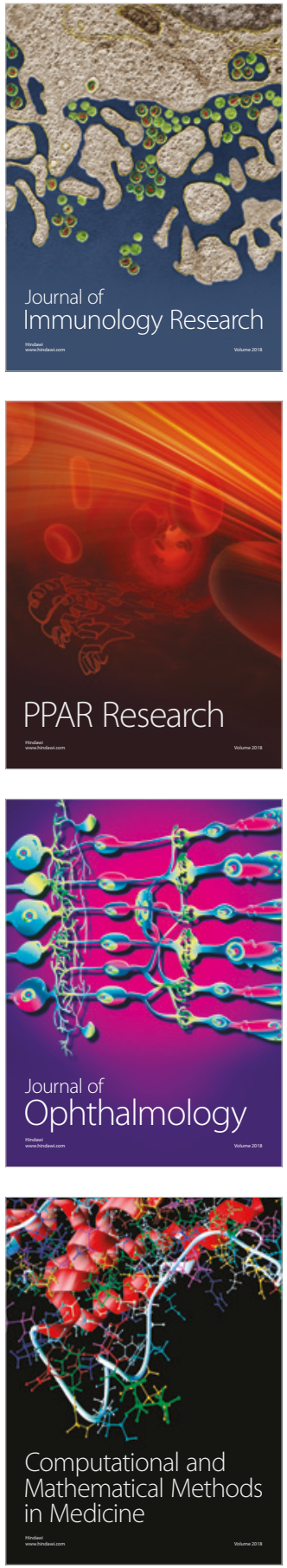

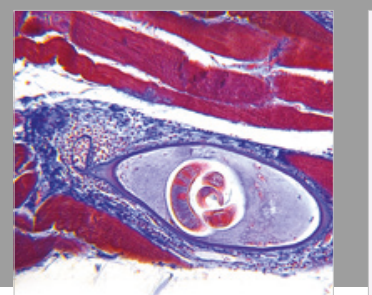

Gastroenterology Research and Practice

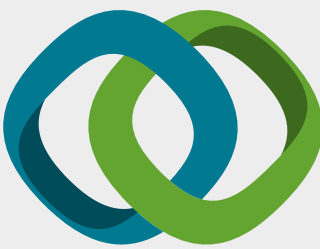

\section{Hindawi}

Submit your manuscripts at

www.hindawi.com
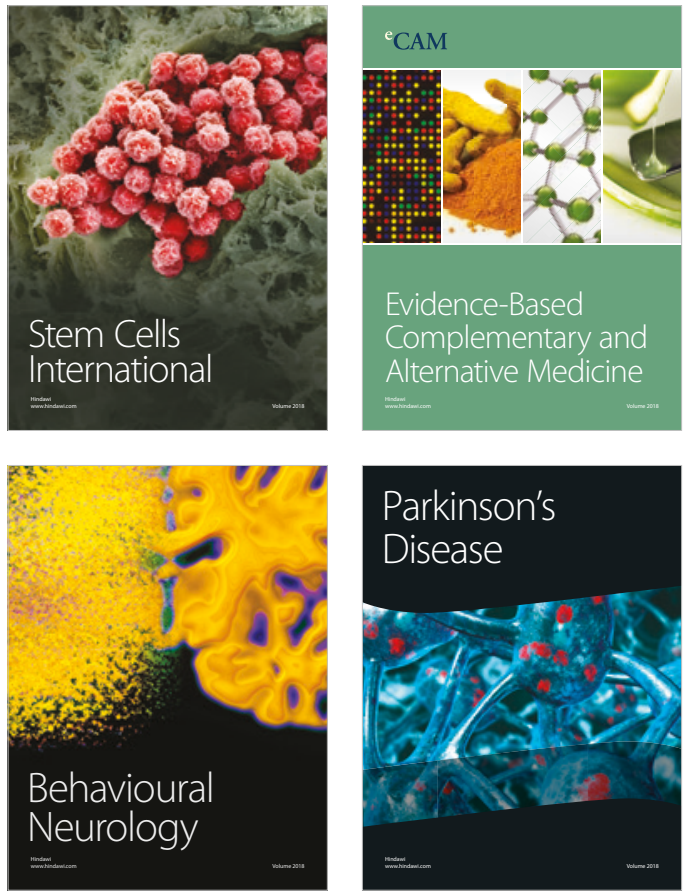

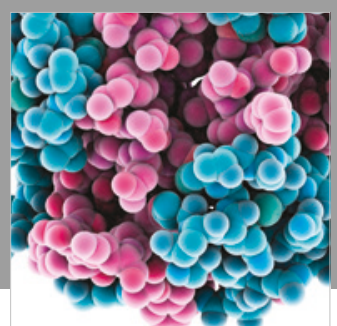

ournal of

Diabetes Research

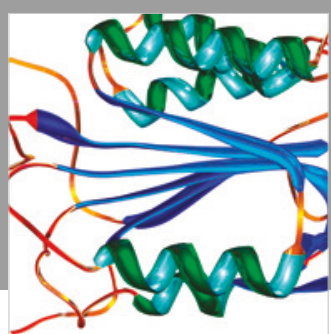

Disease Markers
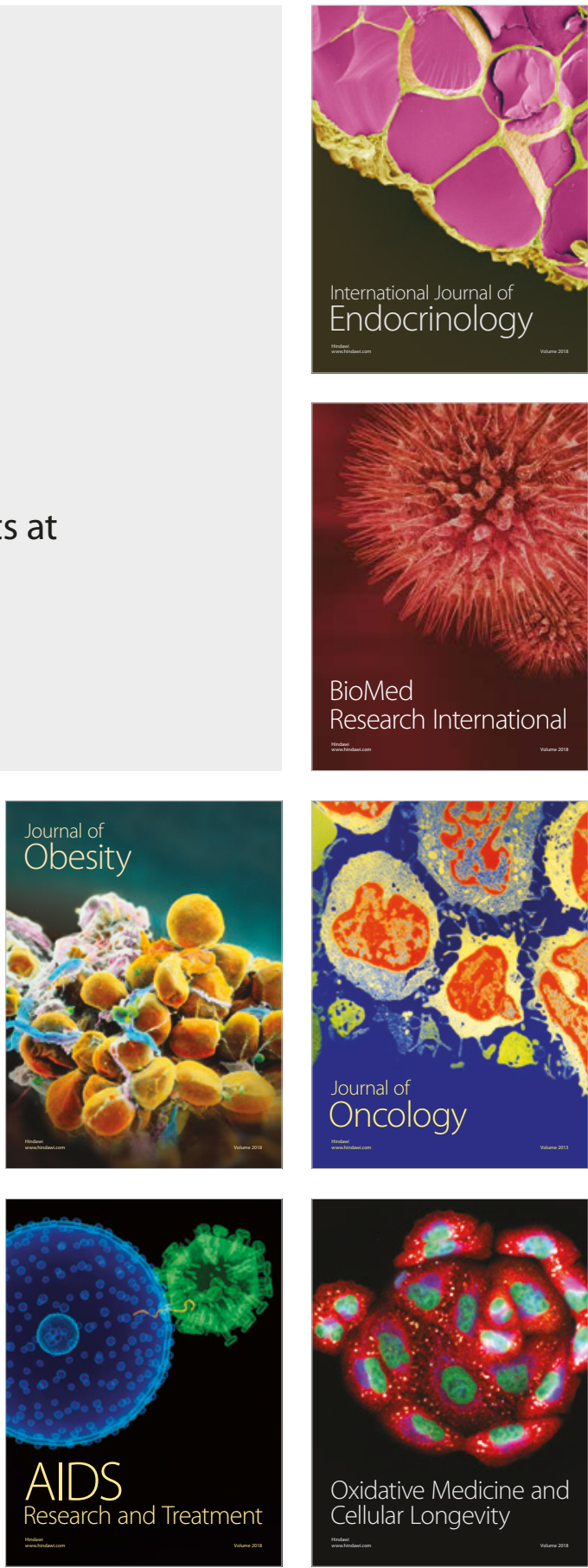